\title{
A Survey on Energy Performance Improvement with Data Aggregation Techniques for WSN
}

\author{
Meethu Abraham \\ School of Electronics Engineering \\ KIIT University, Bhubaneswar, India
}

\author{
Sudhanshu Sekhar Singh \\ School of Electronics Engineering \\ KIIT University, Bhubaneswar, India
}

\begin{abstract}
In the recent advance in science and technology, the communication system has been marching towards quick development. Wireless sensor network is one of the important blocks for communication. The major limiting factors in wireless sensor network are power consumption requirements, life time of network, data integrity and data confidentiality. Data Aggregation is a very critical strategy in wireless sensor network. Data Aggregation is a methodology of gathering and accumulating the message information in a vital effective way, so that life time can be expanded. The paper discusses some standard peculiarity of wireless sensor network associated with the data aggregation and the different approaches of data aggregation.
\end{abstract}

\section{General Terms}

Performances improvement, Network protocols

\section{Keywords}

Wireless sensor network, Energy efficiency, Data aggregation, Base station, Cluster head

\section{INTRODUCTION}

A sensor network is a remote system comprising of spatially appropriated self governing sensors to monitor physical or ecological conditions for examples temperature, sound, and so forth and to pass their information through the system to a principle area. A wireless sensor network comprises of an expansive number of little vitality asset compelled sensor hubs. Each sensor nodes is an accumulation of sensor hardware, microcontrollers, few limits of RAM and program memory, a remote handset and the power supply i.e. battery. The sensor hubs used are small in size and cheap. So that they can be easily embedded in everywhere and create our surrounding perspicacious. With WSN, it is possible to accumulate authentic time data from those places which are dangerous or blocked for wired innovations. WSN can be utilized as a part of numerous courses in modern industrial facility computation. Some of the application is monitoring of equipment, military surveillance and machinery health .WSN can be used for leaking or radioactive monitoring in chemical plant. The two main core challenges in WSN are energy efficiency and scalability [1]. The power consumption in wireless sensor network can be categorized into three domains mainly, i.e. sensing, communication and data processing. The below figure shows the general structure of wireless sensor network in which there are several sensor hubs which are arbitrarily distributed and information about each sensor hub gets accumulated in the base station. Then, with the help of internet user can view information collected by the network with minimum data latency.

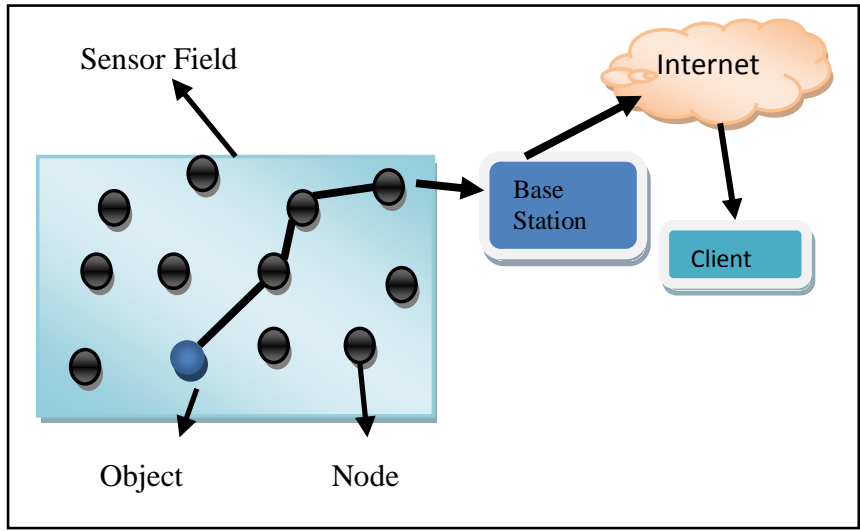

Figure 1: General design of WSN

\section{DATA AGGREGATION}

The primary objective of data aggregation is to eliminate the excess information transmission and in this way upgrade the life time for the remote sensor system. Data Aggregation is one of the fundamental procedures for saving energy in sensor network. It also helps in reducing energy consumption by eliminating redundancy bits.

Data aggregation is a methodology of gathering sensed information utilizing different algorithm techniques. It can also be explained as a technique in which intermediate node in the path receives multiple input data packets and then processes them and transmit as a single packet to the sink or base station. For deploying sensor networks in a hazardous environment, security issues, data confidentiality and integrity in data aggregation become the prime issues of concern [2]. The data transmission in wireless sensor network took place in multi hop fashion. In which each sensor node uses to forward the collected data to the nearby sensor node which is nearer to the base station. The nodes that are placed close to each other can serve the same data which are greatly associated. Because of this spatial relationship, there is a repetition of that information that has to be transmitted to the base station. Data aggregation is a procedure applied to abstract the redundancy which is present.

The general algorithm for data aggregation work as shown in figure 2. The algorithm explains that the sensor nodes senses the data and then aggregate the data using some algorithms. And then the accumulated data are transferred to the base station by choosing the systematic path. Exploiting the merits of data aggregation, one can maximize the robustness and precision of the information and reduce the activity load and moderate the energy of the sensors. On the contrary the drawbacks of the data aggregation is that, the aggregate node or cluster head may be attacked by the dangerous attackers, then the base station cannot guarantee the right aggregated data that has been sent to it. Additionally the number of replicas of that aggregated data can also be send to the base 
station. It enhances the power consumptions at the sensor nodes. The attributes of data aggregation protocols are latency, data rate, energy efficiency, data accuracy and network lifetime.

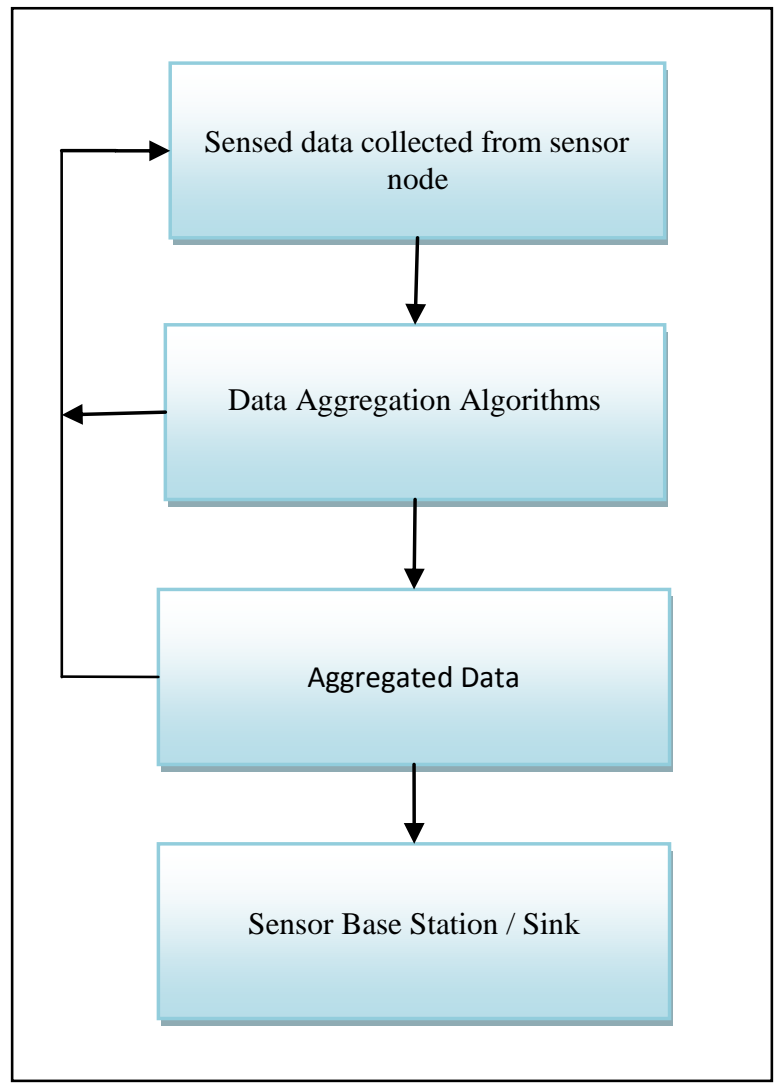

Figure 2: General structure of data aggregation algorithm

\section{DATA AGGREGATION BASED ON ARCHITECTURE}

Data Aggregation is one method which is implemented to evacuate the repetition which is present. Based on the various application and requirement, there are three mainly architecture for data aggregation.

- Centralized Architecture

- Distributed Architecture

- Hierarchal Architecture

\subsection{Centralized Architecture}

The simplest framework of the sensor network is centralized architecture. The each sensor hub faculties information and transmit it to the central node and that central hub have the responsibility of the whole network. The communication in centralized scheme takes place in three stages:

- Gathering Stage : The central node of the network is assumed as the cluster head $(\mathrm{CH})$. The remaining nodes in the cluster transfer the sensed information to the cluster head. This process is known as gathering and a small amount of energy is utilized for this purpose.

- Compression Stage : The information sensed by the sensor hubs in a cluster are associated with each other due to their arrangement. Through the compression at the central node some redundancy can be taken off. The size of the cluster has an impact on the compression efficiency of the cluster.

- Broadcasting Stage : The central node contains the compressed data and it broadcasts that integrated data to remaining nodes in the cluster. The other nodes in the cluster and the access point receives the compressed.

The merit of the above architecture is that it can positively describe the error of the information which is taken by remote sensor network. The prime demerit is the inadaptability to the sensor changes and more workload agitated at a solitary area. The figure 3 demonstrates the centralized architecture.

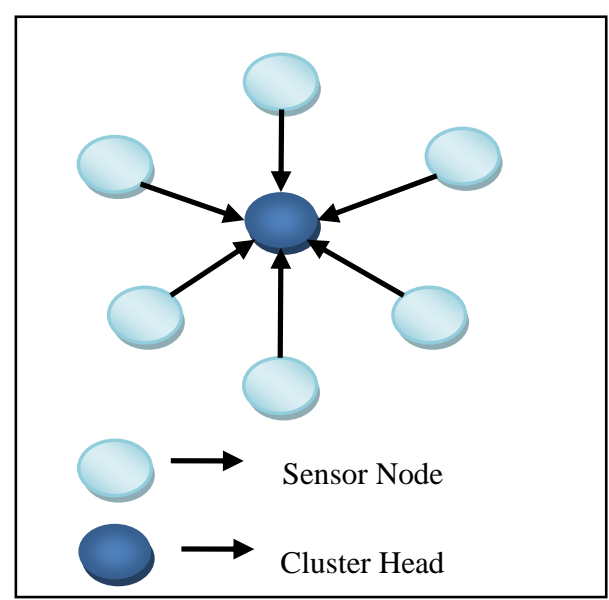

Figure 3: Centralized Architecture of data aggregation

\subsection{Distributed Architecture}

In distributed architecture of data aggregation, there is no centralized node which can make decisions on behalf of all other nodes. The distributed architecture has only two stages:

- $\quad$ Gathering Stage : Every node exchange the sensed information with other nodes by using diverse time period. Furthermore, in this manner every node will have the duplicate of information sensed by all different nodes.

- Compression Stage : In this each node separately collects the data and compress the data aggregated from the above stage.

The merits of this architecture are adaptable and tolerant to expansion or subtraction of sensor nodes. The distributed architecture is shown in figure 4.

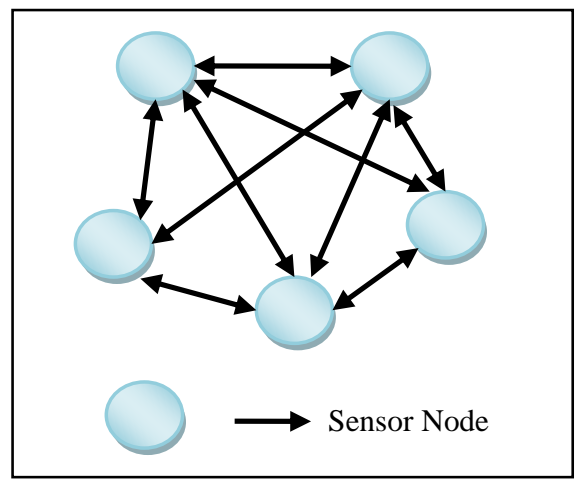

Figure 4: Distributed Architecture of data aggregation 


\subsection{Hierarchical Architecture}

The most important architecture of the wireless sensor network is the hierarchical architecture. Every sensor node is divided into different hierarchical levels. In this architecture, lowest level may contain typical sensor nodes, which are assembled in a topographic region to decrement the transmission potency. The information from individual sensor nodes are transmitted to the data fusion nodes by using the routing algorithm and reducing the power dissipation. The main advantages of hierarchical architecture are the balancing the workload among all the sensor nodes in the wireless sensor network. The hierarchical architecture is shown in figure 5 .

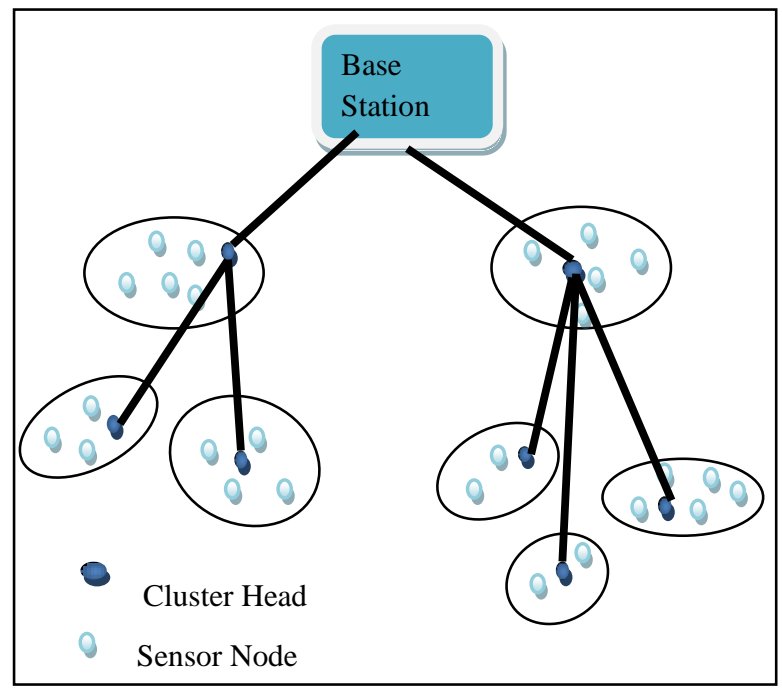

Figure 5.: Hierarchical Architecture of data aggregation

\section{DATA AGGREGATION APPROACHES BASED IN NETWORK}

The design of the sensor network plays an important role in the operation of the different aggregation protocol. They are mentioned below:

\section{Flat Networks \\ 2. Hierarchical Networks}

\subsection{Flat Networks}

In wireless sensor network, flat networks plays very important role. In this each sensor node performs the identical task and contains equal amounts of the battery power. With the help of a data centric routing approach, data aggregation has to be done in the network, where base station usually transmits the query message to the sensor like flooding. In flooding sensor, which have information matching the data packet and response transfer information packet to the base station. Among numerous flat routing protocol like directed diffusion and SPIN are briefly explained below :

\subsubsection{Directed Diffusion (DD)}

Directed diffusion refers as a data centric with an application aware protocol, which is used in wireless sensor network. The network lifetime can be expanded using this protocol. It meets the main requirement of wireless sensor network such as energy efficiency, scalability and robustness. Directed diffusion contains four elements like interest, gradient, data message and reinforcement. An interest is a request which contains data that is sent by base station to the source node.
The gradient is a response link to the neighboring node. Data message are name using attribute value pair. The base station diffuses a query towards the interest area node. Routes are established between the sink and nodes with the help of interest and gradient. In this sink node the message is transmitted to the source node. Every node gets the interest which can be stored for later use. At this point when interest is telecast by network hop by hop , then gradient are setup to draw information from query towards requesting nodes[3].

The above protocol are application particular and can preserve energy by electing the corrected path. The problem with this protocol is the overhead included in recording data along these build the expense of sensor .

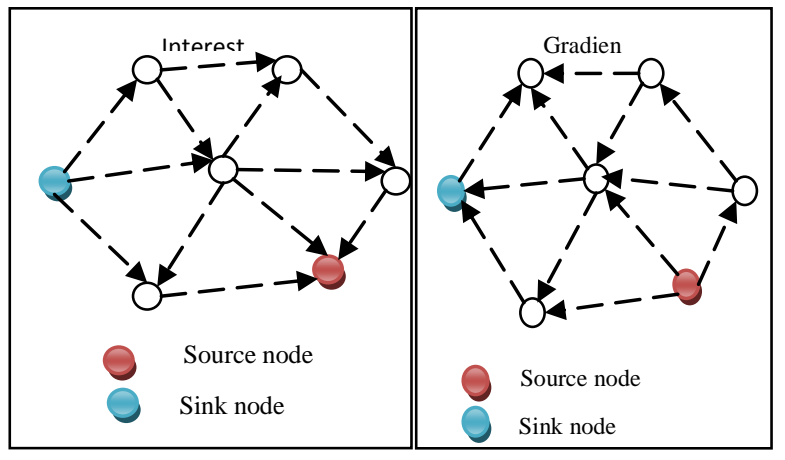

(a)

(b)

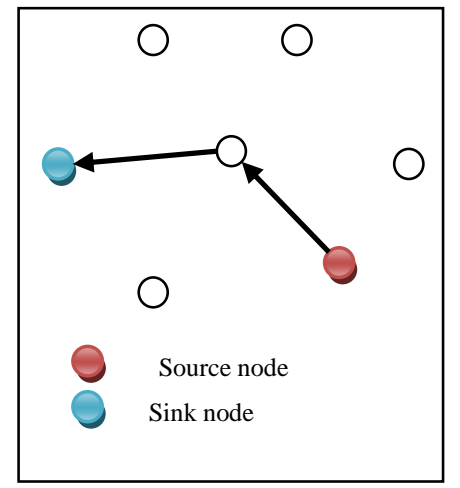

(c)

Figure 6: Directed diffusion stages : (a) Interests propagation stage, (b) Gradients setup stage and (c) Data delivery along reinforcement stage.

\subsubsection{SPIN (Sensor protocol for information via negotiation)}

SPIN is a routing protocol very much data centric and uses data negotiation and resource adaptive algorithms. Employing this, the nodes in system are thought to be base station and neighboring nodes have comparative information. The main conception behind SPIN is to meta data negotiation and resource adaptation instead of full data packet transmission at each node. Node in this network uses high caliber name to depict their gathered data called meta data. The figure-7 indicates the functionality of the SPIN protocol. The meta information is traded among sensor before transmission and after it information advertisement approaches occurs. Consequently maintain a strategic distance from transmission of excess information in the system. While getting the data each node promotes to its neighbor and interested neighbor to get this information according to request message. This meta negotiation resolves the complication of flooding and thus improve the lifetime of the network. In SPIN protocol usually 
three types of messages are transferred are Advertisement $(\mathrm{ADV})$, Request (REQ) and DATA.

$\mathrm{ADV}$ is responsible for advertising the new data and it sends by the sender node towards all the receiver nodes. REQ is used to request the data and is sent by receiver node to the sender node. DATA is the actual message which is sent by the sender node to the receiver node after it receives REQ packet from receiver node.

The two types of SPIN have developed: SPIN1 and SPIN2. SPIN1 is a simple protocol for dismantling data through the network. It uses three stages (ADV- REQ -DATA) handshake approach. It works with first transferring the ADV message by anode to all other node in the network and is respond by REQ message if they want to send the data packet. This protocol allows for data aggregation and works in un configured mode.

SPIN2 is normal SPIN1 protocol when the energy of a node becomes low.

SPIN protocol shows high performance in respect to low cost, complexity and energy efficiency. The topological changes are localized by exploiting the advantages of SPIN since each node is to know only its single hop neighbor.

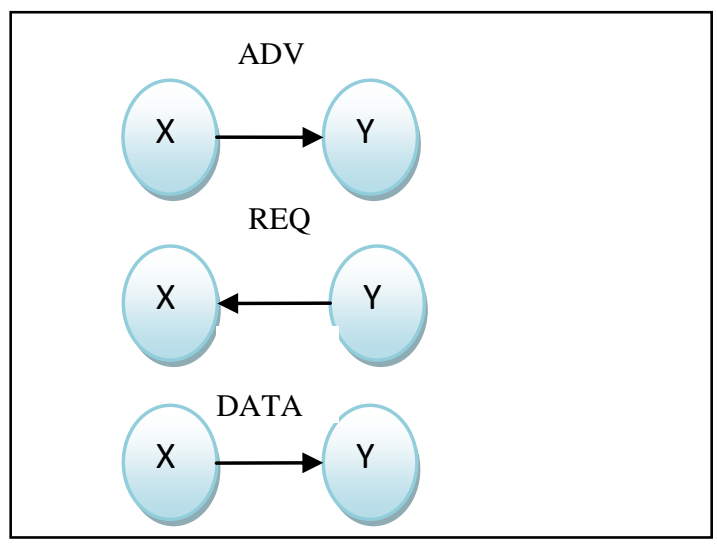

Figure 7. Data transmission in SPIN

\subsection{Hierarchical Networks}

The data aggregation has to be done in hierarchical network with a unique node because of this unique node, the quantity of data packet which transmitted to the base station can be decreased. That is the way this hierarchical system enhances the energy proficiency of the entire system.

The different kind of hierarchical network are described below :

\section{- Data Aggregation based on Cluster \\ - Data Aggregation based on Tree \\ - Data Aggregation based on Chain \\ - Data Aggregation based on Grid}

\subsubsection{Data Aggregation based on Cluster}

Wireless sensor system is a resource constraints that is the reason the sensor hubs cannot specifically broadcast the information to the sink. In clustered network, whole network id divided among clusters. The information is sent through each sensor node to the cluster head or the local aggregator, which aggregates the data packet in its cluster and forward directly to the sink or base station. This clustered scheme helps in saving energy of the sensors and increase the life time of the system. The cluster head can only directly associate with the sink. Figure 8 shows the cluster based organization.

There are few issues additionally included with the clustering in wireless sensor network. The primary issue is the what amount of clusters should be present in a sensor network and the second one is the amount of sensor nodes in a cluster should have it. The third and vital issue is selection methodology of cluster head in a cluster.

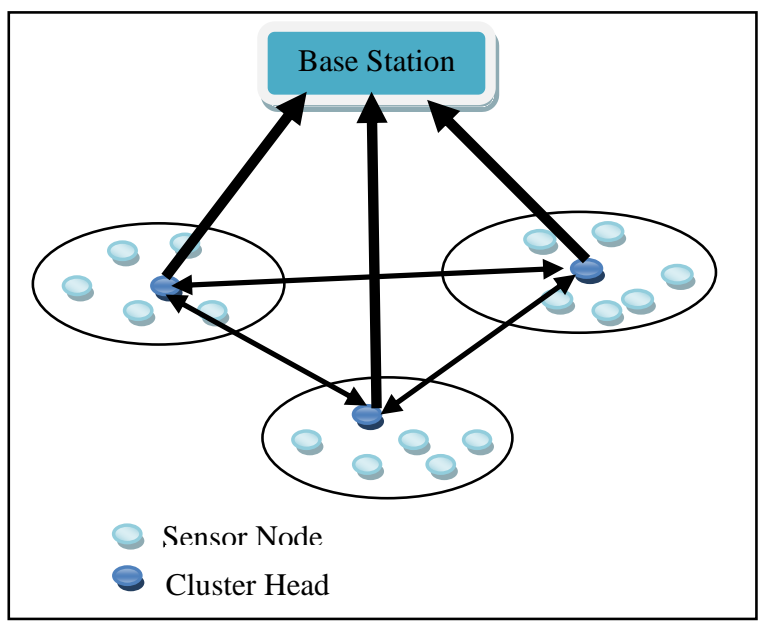

Figure 8: Data aggregation based on Cluster

\subsubsection{LEACH (Low energy adaptive clustering hierarchy)}

LEACH is a self arranging versatile clustering based system and data aggregation protocol. LEACH is the first energy conserving algorithm in wireless sensor network which is proposed for reducing power consumption. The total energy load of the network is distributed randomly among the sensor nodes. In this every sensor hubs are organized into cluster for data accumulation. The clusters are responsible for prolonging the lifetime of WSN system. At this convention, base station is settled and which is spotted far from the node clusters. The WSN environment taken is homogenous and vitality compelled. The cluster head in each cluster send the collected information to the BS. So one can lessen the aggregate number of data that is send to the base station. The data aggregation process is repeatedly executed in the cluster.

The operation of LEACH is divided into rounds and each round is consisting of two phases. The two phases are setup and steady phase. In the setup phase, the network is organized into clusters and cluster head is chosen from sensor hubs at once with certain likelihood. The cluster head advertisement and transmission schedule creation are also involved in this stage. In steady phase, data aggregation process and information transmission process to sink are involved. LEACH has some restrictions because it is considered that all nodes have the same amount of energy and which one be chosen as cluster head. So the Energy consumption of the network is significantly reduced by LEACH protocol by decreasing the communication cost between cluster head and sensor. It is not applicable in larger region [4].

\subsubsection{HEED (Hybrid energy efficient distributive clustering)}

Multi hop clustering algorithm is basic operational mode for HEED in wireless sensor network. HEED extends the basic concept of LEACH by using the remaining energy of the node and node degree for selecting cluster to achieve power balancing. By selecting proper cluster head based on the 
physical distance between the nodes, it improves the efficiency of clustering. In HEED all the node are assumed to be homogenous [5].

HEED has four primary goals and they are 1) increasing the lifetime of network by distributing energy consumption 2) using a constant number of iterations terminating the clustering process 3 ) decreasing control overhead and 4) selecting well efficient cluster head and its cluster. The life time of sensor is limited and there is need to reenergize the sensor network adding more nodes. HEED improves network life time than LEACH randomly select cluster head. Cluster head are selected by two parameters and they are remaining energy of each node and inter cluster communication cost used by node.

\subsubsection{Data Aggregation based on Tree}

The basic approach of data aggregation can be put through an aggregation tree. In this all the all the hubs are sorted out as the tree, with the assistance of the transitional hub and perform information accumulation handle and transmit information from the leaf hub to the parent hub. Each node has a parent node to which data is to be transmitted. The stream of information is from the leaf hub to the base station so aggregation is carried out at the parent hub . The important aspect of the tree based approach is designing of the organized data aggregation tree. The demerit of this approach is that if at any level, there in an occurrence of packet loss, then total data will be lost from each level related to the tree. Figure 9 shows the data aggregation approach based on tree.

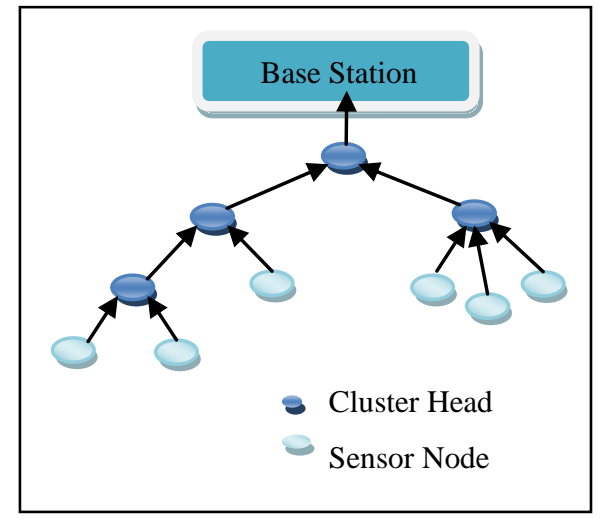

Figure 9: Data aggregation based on Tree

\subsubsection{TAG (Tiny aggregation)}

TAG is basically a generic aggregation service observed in Ad-hoc sensor network. The tiny aggregation service is a data centric protocol and which is also tree based approach. It is basically designed for the monitoring application. In tiny aggregation, information are periodically collected from the all sensor nodes. There are two attributes in tiny aggregation. One is the, it provides simple declarative interface for aggregation and the other one is, its intelligent distributive and executive aggregation operation on sensor network.

Tiny aggregation consists of two phases and they are distributive and collection phase. In distributive phase, aggregator queries are seeking from the network and while collecting aggregated value are routed from root node to parent node. This distribution phase passes a broadcast message from sink in order to organize sensor node into tree. The broadcast message is sent periodically by sink to keep tree structure update.
The main advantage of tiny aggregation is its ability of reducing the frequency of communication for computing aggregate information. The other advantage is that single message transmission is enough for each sensor node regardless the depth in routing tree [6].

\subsubsection{PEDAP (Power efficient data gathering and aggregation protocol)}

Power efficient data gathering and aggregation protocol is a spanning tree based approach protocol. The prime objective of PEDAP is to maximize the life time of the WSN system. The lifetime of the network can be enhanced in term of number of rounds. The aggregated data are transmitted from sensor node to the sink in every round. The algorithm Prim's minimum spanning tree is used to compute routing path with sink as root.

The residual energy of the node is considered to balance a specified node among all the nodes. The global knowledge of network and location of all nodes are required for power efficient data accumulation and aggregation protocol to operate smoothly. It operates centrally allowing base station to compute the routing information.

\subsubsection{Data Aggregation based on Chain}

In chain based approach, each sensor node forward its data to its closest neighbor. For the data aggregation the sensors have been structured in the form of linear chain and using greedy algorithm a chain can be formed by the sensor nodes. While going for formation of greedy chain it expect that all the nodes have full knowledge about the system. The most distant node from the sink begins The chain development is started by the farthest node and at each one stage closest neighbor is chosen as successor of the chain. In data aggregation round each hubs receives the information packet from neighbor, and aggregate the information its own and sends the aggregated information to the other hub which is closer to it, like this way process will be go on. The leader node in this chain based approach are homogenous to the cluster head and $\mathrm{CH}$ sends the aggregated :data to the sink. Figure10 shows the data aggregation based on chain approach Some of the chain based protocol are PEGASIS and CREEC are explained briefly below :

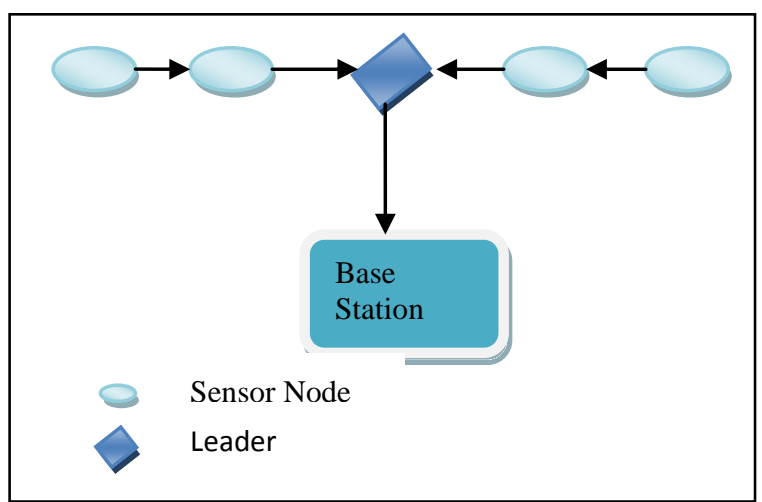

Figure 10: Chain based data aggregation

\subsubsection{PEGASIS (Power efficient gathering sensor information system)}

Another chain based energy saving data aggregation protocol PEGASIS, which is predicated on the LEACH base. Here, each node has the knowledge about all other node and they directly transmit information to the base station. The same amount of energy is available on all the sensor node and they will get die at the same time. 
By using greedy algorithm they can construct chain because each sensor node have detailed knowledge about the wireless network. It is possible to create the chain by a node which is far away from base station. Each node transmit and receive the data to its closest neighbor. Each node use signal strength to determine the nearest neighbor node and calculate the distance from neighbor. The hub passes data through fasten to leader from the either side. In each round arbitrarily pick up node called leader transmit aggregated data to base station. At last the leader sent the aggregate data to base station [7]

PEGASIS outperform LEACH with substantial reduction of the overhead dynamic cluster information, along with the distance and limit of transmission. The demerit of this protocol is that each hub requires overall information of the network.

\subsubsection{CREEC (Chain routing with even energy consumption)}

CREEC attains a larger average life time using increasing the fairness of energy distribution and minimize the total energy consumption. CREEC uses a feedback mechanism of every distribution. In throwing schedule, base station calculates the number of throw to be assigned to any node. The throwing energy can be unified by any node. For chain establishment, there are three steps in every super round by base station to generate a chain

The first step is to calculate the cumulative forward energy by base station and arrange all the node according to the decreasing order of cumulative forward energy and then categorized by three node level. The two most depleted nodes in the list belong to level -1 , the next belong to the level -2 and the remaining belong to the level-3 node. The level 1 nodes are belonged to two leaf node in chain.

In the second step, it generates chain using Kruskal's MST algorithm. In this shortest path is selected and is added to every chain one by one until no more selection is possible. In the last step, the chain length is reduced by a link tramming algorithm called link swap. The chain is updated in every round and build new chain to save energy at depleted node [8].

\subsubsection{Data Aggregation based on Grid}

The data aggregation approach based on a grid is applied to a fixed region of a sensor network in which a set of sensor is considered as the data aggregator. The sensor in a grid sends the information packet specifically to the grid aggregator. All sensors present in a grid do not communicate with each other directly. The data aggregation based on the grid is same as that of in- network aggregation. There are two major differences, i.e. sensor in grid communicate with neighbor node. The hub in a grid can consider as the part of aggregator hub in term of round until it finish. This is very much similar to fixed cluster head based data aggregation.

In network aggregation the most vital data containing sensor node aggregates the data packet and forward it to the base station. Each hub sends its signal to its neighboring hubs. At the point when the neighbor has higher signal it ceases there transmitting an information packet. When the neighbor nodes transmit the information packet to the maximum signal strength node it will be elected as the data aggregator. Thus the in-network aggregation is best acceptable for those environment which is highly localized.

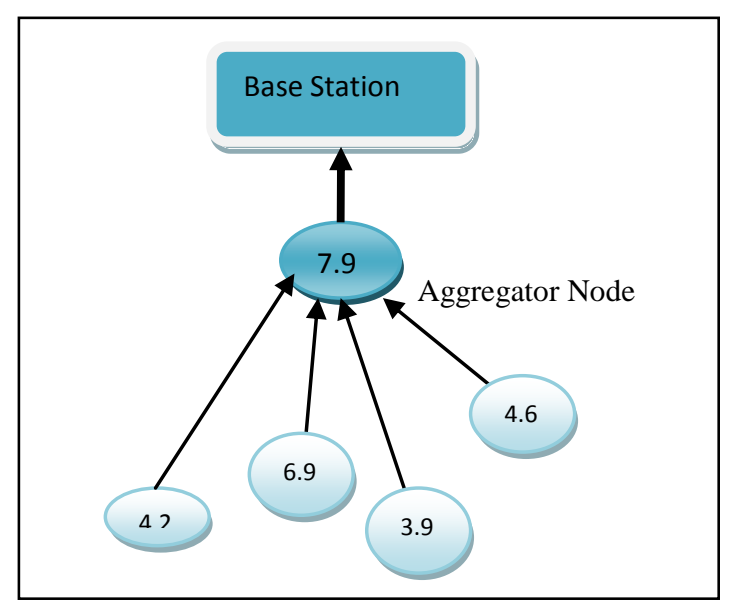

Figure 11: Data aggregation based on Grid

Table 1. Comparative study of various data aggregation techniques

\begin{tabular}{|l|l|l|l|l|}
\hline Protocols & Type & $\begin{array}{l}\text { Energy } \\
\text { Consumption }\end{array}$ & Aggregation & Scalability \\
\hline LEACH & $\begin{array}{l}\text { Cluster } \\
\text { based }\end{array}$ & High & Yes & Good \\
\hline HEED & $\begin{array}{l}\text { Cluster } \\
\text { based }\end{array}$ & Moderate & Yes & Limited \\
\hline PEDAP & $\begin{array}{l}\text { Tree } \\
\text { based } \\
\text { based }\end{array}$ & Low & Yes & Good \\
\hline PEGASIS & $\begin{array}{l}\text { Chain } \\
\text { based }\end{array}$ & High & No & Good \\
\hline CREEC & $\begin{array}{l}\text { Chain } \\
\text { based }\end{array}$ & Low & Yes & Limited \\
\hline DPIN & $\begin{array}{l}\text { Flat } \\
\text { Network } \\
\text { Network }\end{array}$ & Low & Yes & Limited \\
\hline
\end{tabular}




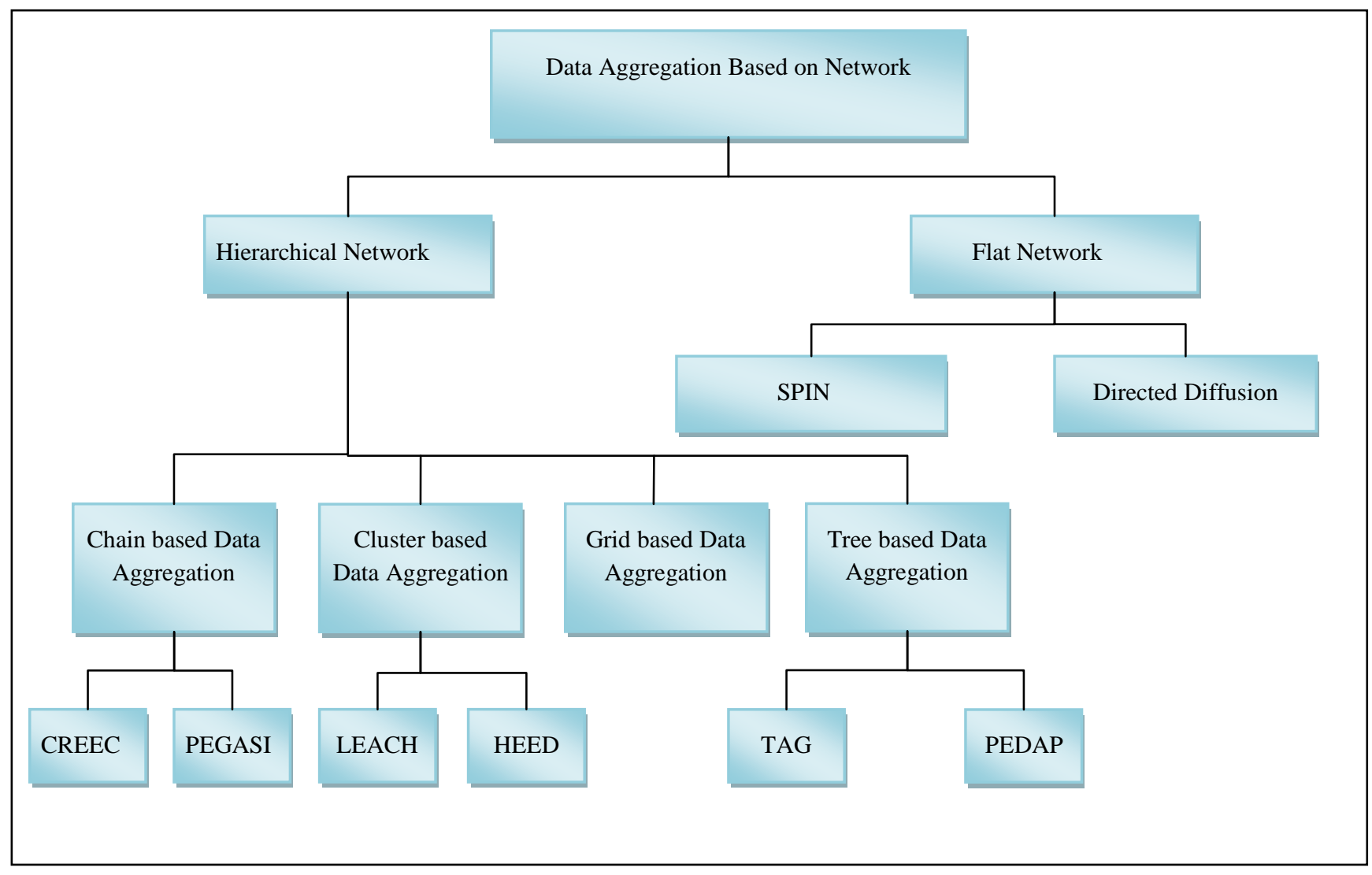

Figure 12: General structure of network based data aggregation

\section{CONCLUSION}

In the present era of communication engineering and technology, the area of wireless sensor networking has received attention among the researches. Data Aggregation is one of the critical strategy for improving the life time and tackle the asset compelled issues in wireless sensor network. This paper describes thoroughly the wireless sensor system, approaches of data aggregation, various data aggregation techniques and their comparison between them. The performance measurement techniques of data aggregation in wireless sensor networks for some vital attributes such as network life time, data latency, data accuracy and energy consumption are surveyed and various techniques and protocols used for enhancement of the performance are observed.

\section{ACKNOWLEDGMENTS}

The authors appreciated the support provided by school of Electronics Engineering, KIIT University, Bhubaneswar for the technical assistances.

\section{REFERENCES}

[1] S. Mishra, H. Thakkar, " Features of WSN and Data Aggregation techniques in WSN : A Survey", International Journal of Engineering and Innovative Technology, volume 1, April ,2011.

[2] Nandini.S.Patil, Prof P.R.Patil, "Data Aggregation in Wireless sensor network", International conference on computational Intelligence and computing network, IEEE 2010.

[3] C. Intangonwiwat, R.Govindan, D.Estrin, "Directed Diffusion : A scalable and robust communication paradigm for sensor network", proceedings of ACM mobile communication, Boston, March 2000.

[4] W.Hienzelman, A.Chandakasa, H.Balakrishnan, "Energy efficient communication protocol for wireless micro sensor network", Proceedings of 33rd Annual Hawaii, international conference on system science, Big Island, USA, January 2000.

[5] Ossama Younis, Sonia Fahmu, "Distributed clustering in adhoc sensor networks : A Hybrid energy efficient approach", Proceedings of IEEE IFOCOM ,Hongkong, volume 1, 2004.

[6] Samuel Madden, Michael j. Franklin, Joseph M Hellerstein ," TAG : a Tiny Aggregation service for adhoc networks", 5th Annual Symposium on Operating Systems Design and Implementation (OSDI). December, 2002

[7] Stephanie Lmdsey, Cauligis Raghavendra," PEGASIS : Power efficient gathering in sensor information system".

[8] Jisoo Shinand, Changjin Suh, " CREEC : Chain routing with even energy consumption", IEEE communications and networks, 2011.

[9] V.Akila, Dr.T.Sheela, "Overview of data aggregation based routing protocols in wireless sensor networks", International Journal of Emerging Technology and Advanced Engineering, volume 3, special issue 1, January 2013.

[10] Kiran Maraiya, Kamal Kant, Nitin Gupta, "Wireless sensor network : A review on data aggregation", International Journal of Scientific and Engineering Research , volume 2, Issue 4, April 2011. 
[11] Kavitha.M , Madhuvarthana.L, Monika.D, Preethi.M.J, " Survey on different data aggregation technique in wireless sensor network", International Journal of Advanced information Science and Technology,ISSN:2319:2682, volume 18, October 2013.

[12] Shio Kumar Singh, MP Singh, DK Singh, "Routing Protocols in Wireless sensor networks : A survey", International Journal of computer science and Engineering survey, volume 1, Issue 2, November 2010.

[13] Subhajit Pal, Debnath Bhattachariya, Geetam Tomar, Tai-hoon Kin, "wireless sensor network and its routing protocols : A comparative study". International conference on computational Intelligence and computing network, IEEE 2010.

[14] Gunti Spandan, Archana Patel, CR Manjunath, Nagaraj GS, "Data aggregation protocols in wireless sensor networks", International Journal of computational Engineering Research, volume 3, Issue 5, May 2013.

[15] Priyav Ujawe, Simran Khiani, "Review on data aggregation techniques for energy efficient in wireless sensor network", International Journal of Emerging Technology and Advanced Engineering, volume 4, issue 7, July 2014. 